- Case Report

\title{
Transfusion Related Acute Lung Injury after Cesarean Section in a Patient with HELLP Syndrome
}

Kyoung Min Moon, Min Soo Han*, Ch’ang Bum Rim, So Ri Kim, Sang Ho Shin, Min Seok Kang, Jun Ho Lee, Jihye Kim, Sang II Kim

Department of Internal Medicine, Eulji University School of Medicine, Daejeon, Korea

Transfusion-related acute lung injury (TRALI) is a serious adverse reaction of transfusion, and presents as hypoxemia and non-cardiogenic pulmonary edema within 6 hours of transfusion. A 14-year-old primigravida woman at 34 weeks of gestation presented with upper abdominal pain without dyspnea. Because she showed the syndrome of HELLP (hemolysis, elevated liver enzymes, and low platelet count), an emergency cesarean section delivery was performed, and blood was transfused. In the case of such patients, clinicians should closely observe the patient's condition at least during the 6 hours while the patient receives blood transfusion, and should suspect TRALI if the patient complains of respiratory symptoms such as dyspnea. Furthermore, echocardiography should be performed to distinguish between the different types of transfusion-related adverse reactions.

Keywords: Acute Lung Injury; HELLP Syndrome; Blood Transfusion; Cesarean Section 


\section{INTRODUCTION}

The syndrome of hemolysis, elevated liver enzymes, and low platelet count (HELLP) is rare and occurs in $0.2 \%-0.6 \%$ of pregnant women. Exacerbation of the HELLP syndrome renders the patient at a high risk for morbidity and mortality. ${ }^{1)}$ In such cases, termination of pregnancy as early as possible is deemed appropriate. In the case of an emergency cesarean section delivery, the patient is highly likely to receive blood transfusion, and eventually develop a serious adverse reaction such as transfusion-related acute lung injury (TRALI). ${ }^{2}$ Herein, we report the case of a 14-year-old patient with the HELLP syndrome who developed TRALI after cesarean section delivery.

\section{CASE REPORT}

A 14-year-old primigravida pregnant woman at 34 weeks of gestation was hospitalized in the division of gastroenterology, Eulji University Hospital, because she complained of epigastric and right upper-quadrant pain without dyspnea. Her vital signs were as follows: blood pressure, 130/80 mm Hg; pulse rate, 94/ min; respiratory rate, $20 / \mathrm{min}$; and body temperature, $36.9^{\circ} \mathrm{C}$. The findings of her laboratory examination were as follows: proteinuria, grade $4+$; platelet count, $1.9 \times 10^{3} / \mu \mathrm{L}$; lactate dehydrogenase, $999 \mathrm{U} / \mathrm{L}$; hemoglobin, $10.6 \mathrm{~g} / \mathrm{dL}$; total bilirubin, 1.5 $\mathrm{mg} / \mathrm{dL}$; aspartate transaminase concentration, $182 \mathrm{U} / \mathrm{L}$; and alanine transaminase, $186 \mathrm{U} / \mathrm{L}$. On the first hospital day, she was diagnosed with the HELLP syndrome (hemolysis, elevated liver enzymes, and low platelet count). She was transfused 13 units of platelet concentrate, 4 units of fresh frozen plasma, 1 unit of apheresis platelet concentrate, and 2 units of leukocytefiltered packed red blood cells before the operation. She was then transferred to the department of obstetrics and gynecology, where she underwent an emergency cesarean section delivery. At 6 hours after the transfusion, she developed dyspnea, and her body temperature elevated to $37.7^{\circ} \mathrm{C}$. Cyanosis on her fingertips and lips was noted. The arterial blood-gas analysis showed hypoxemia: arterial oxygen tension $\left(\mathrm{PaO}_{2}\right), 37 \mathrm{~mm} \mathrm{Hg}$; arterial carbon dioxide tension $\left(\mathrm{PaCO}_{2}\right), 38 \mathrm{~mm} \mathrm{Hg}$; and arterial oxygen saturation $\left(\mathrm{SaO}_{2}\right), 65 \%$. Her chest radiography showed bilateral lung infiltrations (Figure 1A). Computed tomography scan showed diffuse consolidation with multifocal groundglass opacity in both the lungs (Figure 2). Transthoracic echocardiography (TTE) showed that the size and systolic function of the left ventricle were normal without regional wall motion abnormality. She completely recovered with supplemental oxygen in 2 days. Chest radiography conducted 2 weeks after the transfusion showed no active lung lesion (Figure 1B). She recovered without any other respiratory disorder such as dyspnea.

\section{DISCUSSION}

TRALI is the third leading cause of transfusion-associated mortality; however, it is often underdiagnosed and underreported. ${ }^{3)}$
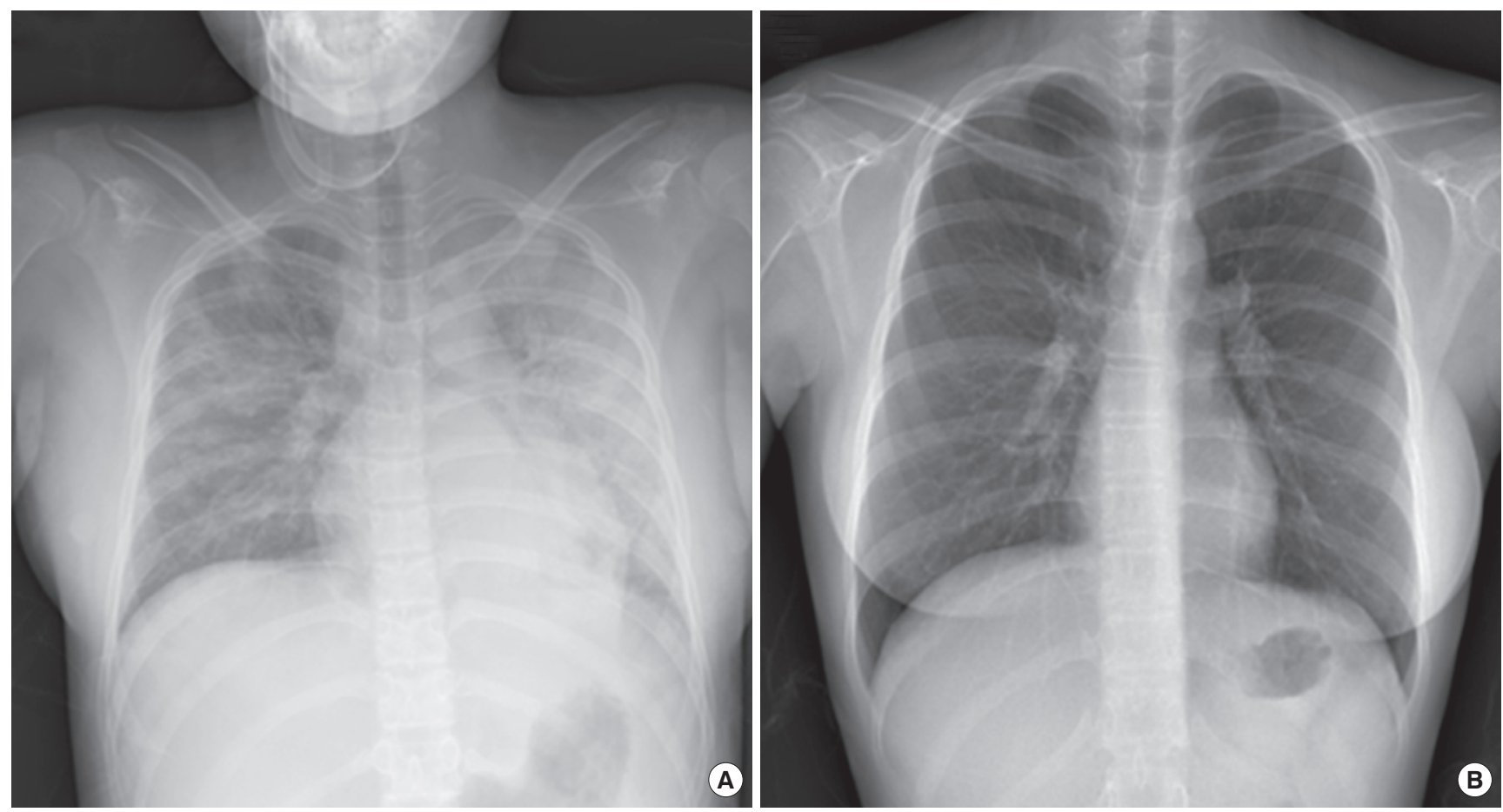

Figure 1. (A) Chest radiography, conducted 2 hours after transfusion, showed bilateral lung infiltrations. (B) Chest radiography, conducted 2 weeks after transfusion, showed no active lung lesion. 


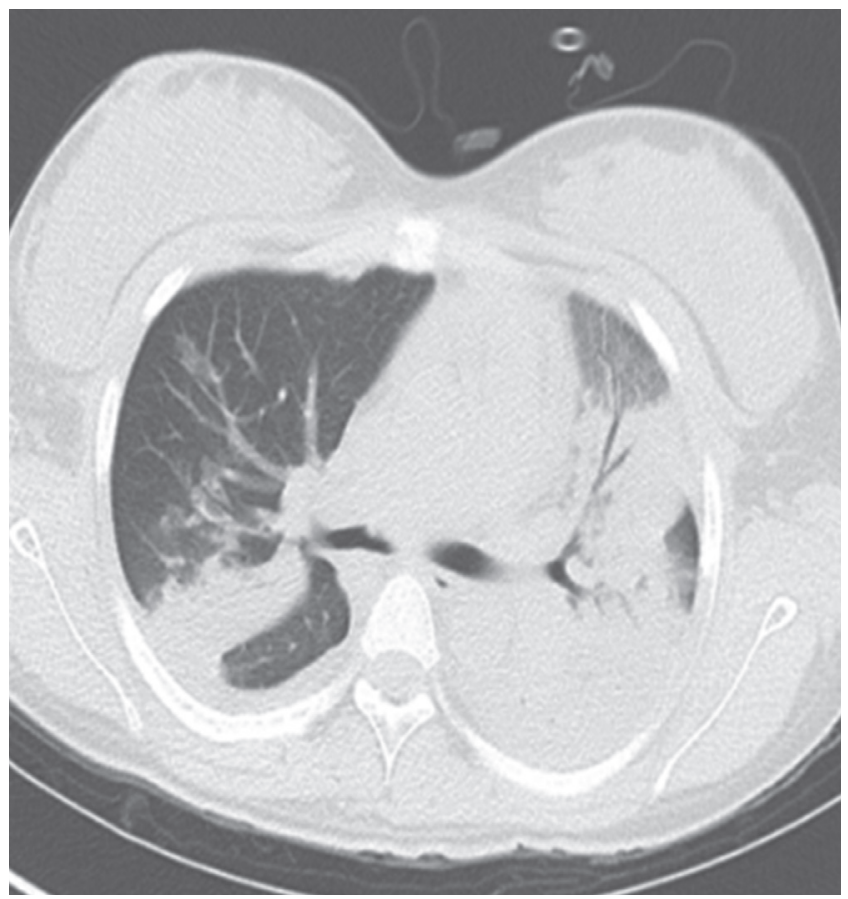

Figure 2. Enhanced chest computed tomography scan showed diffuse consolidation with multifocal ground-glass opacity in both the lungs.

The lack of recognition of this injury can result in inappropriate treatment, as well as failure to report the reaction to the transfusion services and the blood-collection facility. ${ }^{3)}$ Unfortunately, the signs and symptoms associated with TRALI can easily be confused with those of other conditions, including transfusionassociated circulatory fluid overload (TACO), pneumonia, and acute respiratory distress syndrome. The key point in the differentiation of TRALI from the other types of pulmonary edema is that the pulmonary edema in TRALI is non-cardiogenic and that the patients do not show volume overload. The treatment for TRALI involves maintenance of the hemodynamic status and application of ventilatory support. ${ }^{3)}$ The differential diagnosis of TRALI is important, because aggressive diuretic therapy can result in further hypotension, shock, and death.

Especially, the probability of transfusion is high when a patient with the HELLP syndrome is scheduled for an emergency cesarean section delivery. Erdemoglu et al. ${ }^{4)}$ reported that $46.03 \%$ of patients with the HELLP syndrome required transfusion of blood or blood products.

During or within 6 hours of transfusion, regardless of the amount or blood or blood products transfused, if a patient shows acute-onset dyspnea, clinical differential diagnosis of TRALI and TACO should be first performed. Thereafter, a decision should be taken whether the hemodynamic status should be maintained and ventilatory support applied in the event that TRALI is diagnosed, or diuretic therapy be administered in the event that TACO is diagnosed.

In our case, immediately after the patient developed dyspnea at 6 hours after the transfusion, we performed TTE to distinguish between TRALI and TACO in addition to the arterial blood-gas analysis, chest radiography, and computed tomography. TTE showed that the size and systolic function of the left ventricle were normal without regional wall motion abnormality. Therefore, she was diagnosed with TRALI and administered treatment with supplemental oxygen.

Our case of TRALI after cesarean section delivery in a 14-yearold pregnant woman with the HELLP syndrome is unique. First, the HELLP syndrome is rare and occurs in $0.2 \%-0.6 \%$ of pregnant women. ${ }^{1)}$ In cases where the HELLP syndrome is accompanied by TRALI, the latter condition would be overlooked if not suspected clinically and could result in hypotension or shock if it is misdiagnosed as TACO, followed by administration of aggressive diuretic therapy. However, we closely observed the patient's condition during the blood transfusion and suspected TRALI accompanying the HELLP syndrome on the basis of the results of TTE; furthermore, we maintained the hemodynamic status and provided supplemental oxygen without administration of a diuretic therapy.

Second, mechanical ventilation is important for the treatment of patients with TRALI. Looney et al. ${ }^{5)}$ reported that $91 \%$ of the patients with TRALI were treated with mechanical ventilation, and that $78 \%$ of the patients who developed edema required new mechanical ventilation. However, in our case, the patient completely recovered after she was administered supplemental oxygen without mechanical ventilation.

Third, to our knowledge, a well-documented report on TRALI accompanying the HELLP syndrome is lacking. We searched for reports by using terms related to TRALI and the HELLP syndrome on PubMed, but could not find any well-documented report. Thus, we experienced a unique case of TRALI after cesarean section delivery in a 14-year-old patient with the HELLP syndrome.

Lastly, the patient in our case was not an adult (14 years old). Generally, most pregnant women are more than 18 years old, and our case is rare in that the patient was pregnant at a rather young age. The patient had a history of having been raped. Lieberman et al. ${ }^{6)}$ reported that the majority of patients with TRALI were adults, and that there were no significant differences in the occurrence of TRALI between children and adult patients. Most children who presented with TRALI were either teenagers or less than 1 year old. ${ }^{6}$ In our case, the patient was a teenager, and we suspected TRALI immediately, confirmed the diagnosis, and treated the patient.

In conclusion, clinicians should closely observe the patient's condition at least during the 6 hours while the patient receives blood transfusion, suspect TRALI if the patient complains of respiratory symptoms such as dyspnea, and perform echocardiography to distinguish between the different types of transfusion-related adverse reactions. 


\section{CONFLICT OF INTEREST}

No potential conflict of interest relevant to this article was reported.

\section{REFERENCES}

1. Martin JN Jr, Rose CH, Briery CM. Understanding and managing HELLP syndrome: the integral role of aggressive glucocorticoids for mother and child. Am J Obstet Gynecol 2006;195:914-34.

2. Toy P, Popovsky MA, Abraham E, Ambruso DR, Holness LG, Kopko PM, et al. Transfusion-related acute lung injury: definition and review. Crit
Care Med 2005;33:721-6.

3. Kopko PM, Marshall CS, MacKenzie MR, Holland PV, Popovsky MA. Transfusion-related acute lung injury: report of a clinical look-back investigation. JAMA 2002;287:1968-71.

4. Erdemoglu M, Kuyumcuoglu U, Kale A, Akdeniz N. Factors affecting maternal and perinatal outcomes in HELLP syndrome: evaluation of 126 cases. Clin Exp Obstet Gynecol 2010;37:213-6.

5. Looney MR, Roubinian N, Gajic O, Gropper MA, Hubmayr RD, Lowell CA, et al. Prospective study on the clinical course and outcomes in transfusion-related acute lung injury. Crit Care Med 2014;42:1676-87.

6. Lieberman L, Petraszko T, Yi QL, Hannach B, Skeate R. Transfusion-related lung injury in children: a case series and review of the literature. Transfusion 2014;54:57-64. 